\title{
ON THE MORPHOLOGY OF RECONFIGURABLE HYBRID STRUCTURES BASED ON THE EFFECTIVE 4-BAR MECHANISM
}

\author{
M. MATHEOU ${ }^{1}$, M.C. PHOCAS ${ }^{1}$ \& E.G. CHRISTOFOROU ${ }^{2}$ \\ ${ }^{1}$ Department of Architecture, University of Cyprus, Cyprus. \\ ${ }^{2}$ Department of Electrical and Computer Engineering, University of Cyprus, Cyprus.
}

\begin{abstract}
Reconfigurable systems of hinge-connected beams strengthened through struts and continuous cables, utilize their morphology and cable active members through a synergetic process to enhance structural flexibility, stability and energy efficient transformability. The 'effective 4-bar' concept may be applied for the transformation of planar n-bar systems, using a sequence of 1-DOF motion steps through selectively locking (n-4) joints of the primary members and actuating the cables, in order to adjust the system's joints to the desired values during the motion steps involved. The control system includes only two motion actuators located at the structural supports, as well as brakes installed on each individual joint. It performs the reconfiguration sequences through tensioning of one single cable at a time. A numerical investigation presented in the current paper involves four arch systems of 8, 9, 10 and 11-bar linkages with 60/90, 75/75 and 90/60 cm strut lengths on each side of the systems' circumference. In their initial position, all arch systems have $5.0 \mathrm{~m}$ span and $5.35 \mathrm{~m}$ height following a quasi-vertical ellipsoid shape. The target configuration of the systems with $4.20 \mathrm{~m}$ height corresponds to a quasihorizontal ellipsoid shape. Different reconfiguration sequences are investigated, in order to achieve the target configuration for each system. The comparative numerical analysis refers to the maximum stresses developed in the members and the required brake torques for each transformation. The analysis provides an insight into the hybrid structural morphology and mechanical characteristics of the members for optimal implementation of the reconfiguration approach.
\end{abstract}

Keywords: multiple bars linkage, motion planning, reconfigurable structures.

\section{INTRODUCTION}

Kinetic structures of different typologies and mechanisms have been developed in recent years, primarily in terms of deployable structures, in relation to requirements of temporary environments, aerospace and technological progress [1]. In this frame tensegrity and scissor-like systems have been proposed to provide transformability.

In particular, tensegrity structures, i.e. self-stressed systems composed of tension and compression members, are characterized by their physical properties in providing a holistic and integrated composition of embedded active control, since the members may support or replace sensors and active control, respectively. Also, the specific typology comprises autonomous and self-supported systems in all stages of transformation, from the initial to the target ones, especially in the intermediate ones, while effectively transferring the loading [2]. In principle, the transformation of 'static tensegrities' in kinetic systems, actually in terms of deployable systems, is based on the replacement of compression members with linear pneumatic actuators, or the implementation of tension members with variable length [3-5].

In terms of minimizing the energy consumption in deployable tensegrity systems, dynamic models have been developed for systems with one, two and three degrees-of-freedom (DOF), with consequent simplification of the system through replacement of the tension members with pneumatic actuators and tension members with spring elements. Nevertheless, even if the actuators are locked, the stiffness of the system decreases under external loading [6-9]. 
In addition, control by pneumatic actuators, does not enable the system to have adequate stiffness during the transformation, nor to undergo large transformations [10].

Although structures with embedded actuators in place of primary joints or members are usually designed to use a small number of components to achieve a maximum number of shape adjustments, implementation requires designers to deal with an increase of the structure's weight (depending on the number and characteristics of the actuators used), complex mechanisms and energy-inefficient operation. Further related improvements refer to reducing the number of actuators used through clustering of continuous cables and their actuation at the supports of the structure, as well as the reduction of the self-weight increase due to the otherwise integrated control mechanism. Consequently, a reduction of the energy consumption is possible. In this frame, optimization with regard to embedded active control in tensegrity structures is possible through interconnection of the compression members and implementation of continuous active cables (strut-routed actuation) [11]. A related analytical and experimental study is conducted with a transformable pentagon tensegrity structure in [12].

Other common types of deployable structures include planar and spatial scissor-hinge elements. These have the ability to expand in both horizontal and vertical direction [13-15]. While such structural elements need additional stabilizing members like cables or other locking devices, self-stable structures can be achieved with the application of special geometrical configurations through additional inner scissor-like elements. A considerable advance in the design of such systems was made with the development of a simple angulated element and its geometrical principles $[16,17]$. However, scissor-like elements do not provide extensive flexibility, since their motions are limited between the open and closed states. A novel planar and spatial scissor-hinge mechanism is proposed in $[18,19]$. In general, both tensegrity and scissor-like elements are not capable to achieve multiple shapes without altering the overall system's geometrical size and boundaries.

The methodological approach of this work is summarized as follows: In order to exploit the advantages of reconfigurable buildings, a kinematic mechanism, namely the n-bar linkage, was selected as the basic structural and kinematic element of the proposed system. This mechanism can provide the required versatility for a building to assume diverse forms. A motion planning and control approach was then devised to deal with the kinematic complexity of the specific mechanism and simplify the control requirements. This procedure involves alternative multi-step reconfiguration sequences and an optimal one can be selected based on specific criteria. For the implementation of the approach the basic structure was installed with a system of pulleys and actuation cables to constitute a hybrid system and grant additional structural advantages.

The development of planar reconfigurable hybrid structures with hinge-connected members and continuous cables aims at achieving conceptual simplicity on the structural typology, flexibility with regard to the transformability of the system and reduced energy consumption during reconfigurations. The authors had been engaged in the conceptual development of an adaptable structure of spatial hinge connected beams and three planar secondary members rotated at a relative angle of $120^{\circ}$, on the transverse plane of the primary members [20]. Relevant motion planning principles of such linkage systems have been conceived based on a basic planar 7-bar system, which was experimentally tested in small scale [21]. In parallel, a planar 9-bar hybrid system has been investigated in its static and kinetic behaviour on the basis of a structure, supported and controlled through a secondary system of struts and continuous cables with variable length. Transformation of the system is based on the kinematics of an equivalent 4-bar mechanism, using a sequence of 1-DOF motion steps. Each step includes the selective locking of (n-4) joints of the primary members and actuating the cables, in order 
to adjust the system joints to the desired values during the motion steps involved [22]. In the current paper, the kinematics of four planar arc hinge supported hybrid systems are studied to address influence factors in the systems' behaviour, like number of bars, geometrical, mechanical characteristics of the members and motion sequence followed with regard to a given initial and target configuration. The systems are classified through the number of members i.e. 8-, 9-, 10-, 11-bar linkage, the position of the struts at the joint connection of the primary members, i.e. A: $90 / 60$, B: $75 / 57$, C: $60 / 90$, above and below the neutral axis of the beams respectively, and the pattern followed for the transformation. The overall completion of motion during the transformation is grounded on the synergy of the mechanism composition and the alteration of the cables length. The analysis of the kinetic systems has been conducted with the 2D simulation and analysis software, Working Model 2D.

\section{EFFECTIVE 4-BAR METHOD}

Hinge-connected members compose the kinetic system, prescribing pure rotation, translation and complex motion at the connected members. In general, a planar n-bar mechanism of hinge-connected members has (n-3) degrees of freedom and the motion control is determined by the application of an equal number of actuators. Implementation of the effective 4-bar mechanism in kinetic systems may prevent increase of the overall self-weight of the structure, deformations and erection costs; otherwise the system would become energy inefficient. Main reason for this, is the positioning of a single only actuator, hydraulic or electric, on either of the supports, enabling complete control of the motion with 1-DOF of the respective active mechanism of four hinge-connected members (effective 4-bar -E4B), by selectively locking (n-4) joints, Fig. 1. A group of continuous locked joints defines the effective link. In the case examples that will be investigated, the required geometrical transformations are managed by a control system that handles the operation of two actuators on both supports. In every active mechanism of four hinge-connected members, any joint can be adjusted to the desired value and kept locked in all subsequent steps until completion of the system's transformation. The kinetic structure is defined as a closed chain mechanism, since it is composed of links and joints and two ends supported on the ground.

\subsection{Analysis Models}

Four planar systems comprise the simulation models, differentiated with 8, 9, 10 and 11 hinge-connected members and a secondary system of cables and struts. The initial and final

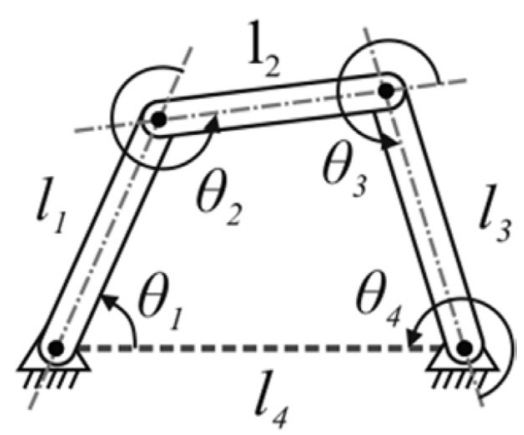

Figure 1: The basic 4-bar linkage mechanism. 


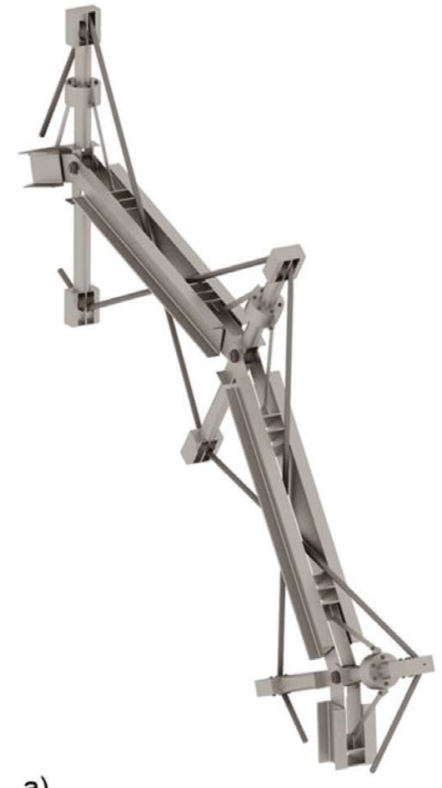

a)

Figure 2: Kinetic structure unit; (a) components design; (b) members' connection with hydraulic brake system.

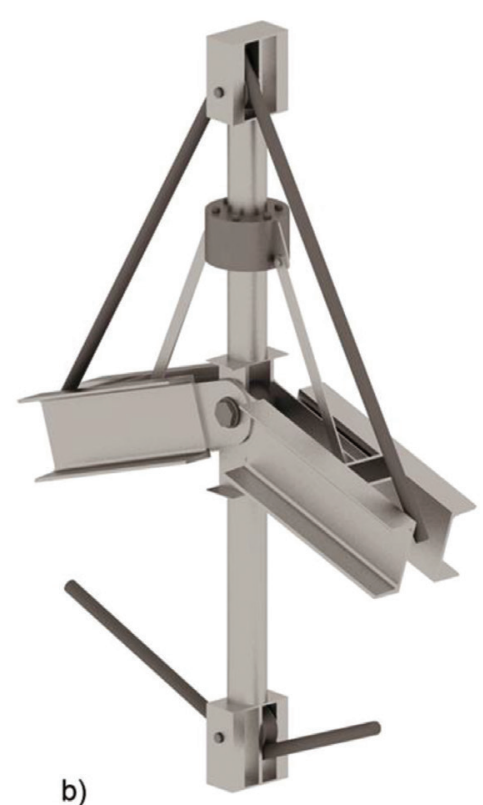

configuration for all systems is defined on the basis of a quasi-ellipsoid form of 5.35 and $4.20 \mathrm{~m}$ height respectively and span of $5.00 \mathrm{~m}$. Adjacent beams are hinge-connected to the continuous struts, Fig. 2. The diagonal cables are continuous with one side connected to a support and the other side to a corresponding actuator. Geometrical characteristics of the kinetic hybrid systems are described as follows: Beams, $2 \times$ UPN 180/150 mm, struts, cross section $60 / 60 / 5 \mathrm{~mm}$, and cables, $\varnothing 20 \mathrm{~mm}$. The mass of the members amounts as follows: Beams in 8-bar linkage of $2 \mathrm{~m}$ length, $88 \mathrm{~kg}$ each, in 9-bar linkage of $1.75 \mathrm{~m}, 77 \mathrm{~kg}$ each, in 10-bar linkage of $1.55 \mathrm{~m}$ length, $68.2 \mathrm{~kg}$ each, in 11-bar linkage of $1.40 \mathrm{~m}$ length, $61.6 \mathrm{~kg}$ each, struts of $1.5 \mathrm{~m}$ length, $13 \mathrm{~kg}$ each, and cables of approximately $20 \mathrm{~m}$ length, $178 \mathrm{~kg}$ each.

The transfer of motion is due to the respective adjustment of the cables' length by the actuators at the supports and the hydraulic linear brakes system positioned axially at the struts and connected over diagonals to the beams. Control of rotations of the members is achieved through the hydraulic linear brakes' system. This provides possibility of adjustment of the connection angle, in avoiding oscillation of the members and achieving effective position of the struts in equally dividing each connection in any stage of transformation. Figure 3 presents the effective 4-bar that corresponds to a particular selection of locked joints of the n-bar systems under investigation. The E4B consists of the input $\left(l_{1}\right)$, the connecting $\left(l_{2}\right)$ and the output member $\left(1_{3}\right)$. Each member of the linkage, is called connecting member (link 1, link 2, . link 9). The position of the mechanism is defined by the vector $\theta \in T_{n}$ that includes angles $\theta_{1}, \theta_{2}, \ldots$ $\theta_{n}$. The angles of a corresponding E4B are collected in vector $q \in T_{n}$ that includes $q_{1}, \ldots q_{4}$. These four unlocked joint angles compose the E4B, which has 1-DOF. When the connecting member 1 activates the angle of joint $\theta_{1}$, then an angle $\mathrm{q}$ of the E4B is adjusted. The initial and target configurations of the four systems are shown in Fig. $3 \mathrm{c}$ and d, respectively. 

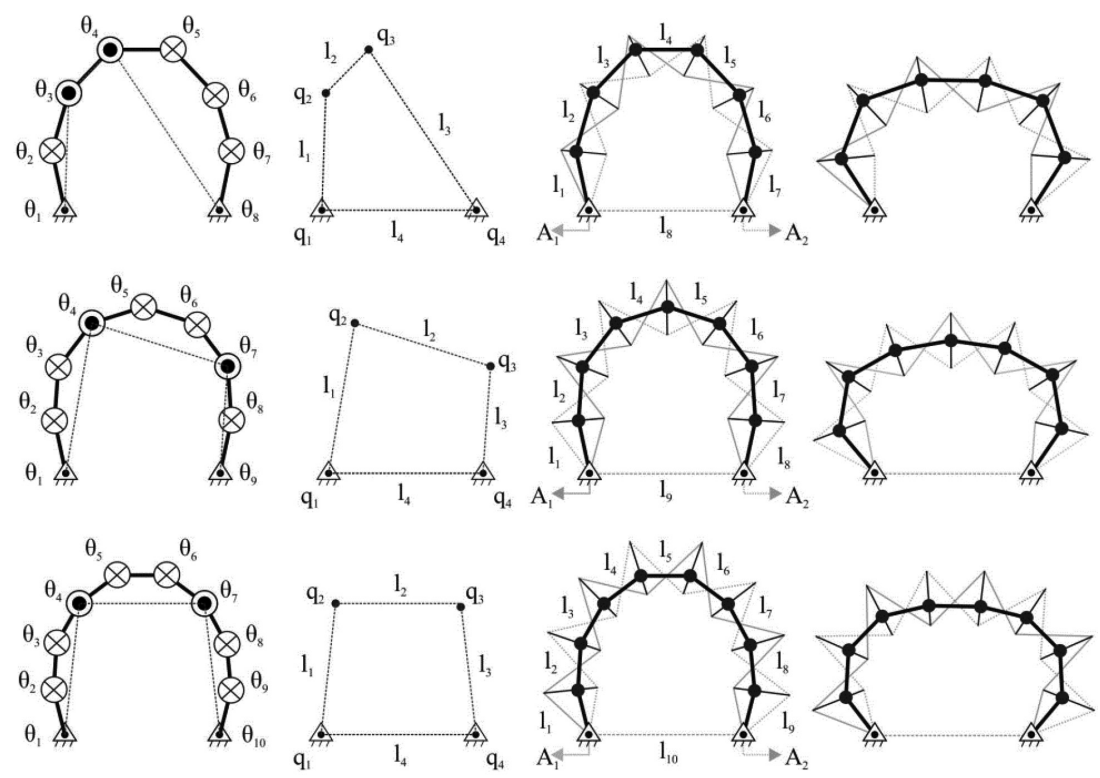

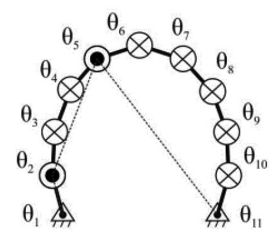

a)

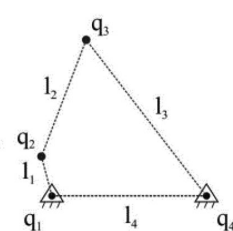

b)

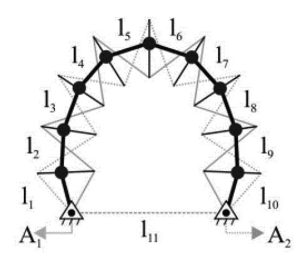

c)

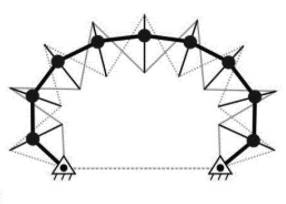

d)

Figure 3: The effective 4-bar concept for the 8-, 9-, 10-, 11-bar systems ( $\otimes$ : locked joint, $\odot$ : unlocked joint, $\Delta$ : pivoted-to-the-ground joint) applied at a transformation step between the initial and target positions of the systems; (a) n-bar linkage; (b) effective 4-bar; (c) initial system configuration; (d) target system configuration.

Intermediate reconfiguration steps of the systems determine the motion patterns of the sequences. By using the available actuator for the respective series of cables, any joint angle of the E4B can be adjusted to the desired value and then it remains locked in the further steps of motion. For each step of a controlled sequence, a different E4B is defined and a joint angle is adjusted. The final E4B is used to adjust the four remaining joints. Supposing that during each step a joint is completely adjusted, it means that a complete reconfiguration of the mechanism of hinge-connected members requires in total (n-3) steps. For each motion step it is necessary that the currently adjusted joint is included in the E4B. The active joint for every effective 4-bar mechanism and the achievement of the complete position of the system is controlled respectively through the hydraulic linear brakes system positioned at the struts and the two actuators at the ground supports. The dual operation of the cables is significant for the kinetic structure. The cable activates any transformation process, and maintains the system stable under loading. The cables to be tensioned in any step define the direction of the system's transformation. Pulleys located at the upper and lower strut points transfer the cables motion. 


\section{MOTION PLANNING}

The transformations of the systems are based on application of the E4B that requires planning of the motion for the systems under investigation. The initial and target position of the four systems with 8-, 9-, 10- and 11-bars, serves the plurality of feasible geometrical transformations. The initial configuration of the 8-, 9-, 10- and 11-bar system is defined by $\theta_{i}=[102$, $152,154,133,133,154,152,102]^{\mathrm{T}},[102,164,147,144,147,144,147,164,102]^{\mathrm{T}},[104$, $162,155,155,143,143,155,155,162,104]^{\mathrm{T}}$ and $[106,161,163,158,143,159,143,158$, $163,161,106]^{\mathrm{T}}$ degrees, respectively. The target configuration is defined by $\theta_{\mathrm{f}}=[123,126$, $130,161,161,130,126,123]^{\mathrm{T}},[128,131,129,160,162,160,129,131,128]^{\mathrm{T}},[129,137$, $138,148,167,167,148,138,137,129]^{\mathrm{T}}$ and $[138,131,142,154,161,166,161,154,142$, $131,138]^{\mathrm{T}}$ degrees, respectively. Many alternative sequences will be possible but their number is reduced after construction kinetic constraints are considered.

The complete reconfiguration of the systems requires specific adjustments of the joint angles and variation of the cables length for every n-bar composition. Practically, the simultaneous adjustment of all joint angles is not possible and a planning methodology of a linear procedure of multiple steps is proposed with the aim to adjust one specific joint angle in every step. The organization of the sequences is based on the motion of the simulated systems and the E4B principle, requiring (n-4) joints unlocked. It is supposed that during motion, no inertial forces are developed in the members of the systems, due to the slow motions involved. A primary motion constraint is associated with the singular configurations of the resulting E4B systems, as analysed in [21]. In addition, another constraint results from the cables-struts system. While the mechanism assumes a position where the cable that passes through a strut is becoming straight, the ability of the system to adjust the corresponding angle is weakened. In this respect an inclination limit (e.g. $175^{\circ}$ ) needs to apply for any consecutive members whose angle needs to be adjusted.

Based on the cables' operation, the sequences are organized in two consecutive unlocked joints and/or two continuous locked joints among the unlocked ones. In this way the cable actions on the joints do not compete with each other, i.e. produced torques do not tend to move the system towards a different direction. All sequences that will be considered are controlled based on a clockwise and vice versa, on a counter clockwise rotation adjustment order. Respective criteria are as follows:

- Control with two consecutive unlocked joints that shift one position (clockwise rotation adjustment) in each step [sequence pattern 1].

- Control with two consecutive locked joints that shift one position (counter clockwise rotation adjustment) in each step [sequence pattern 2].

- Control based on the smallest joint angle difference between initial and target state, while preventing flattening of the members and cables, and following gravity [sequence pattern 3].

- Control initiated with adjustment of two joint angles on the supports followed by the criteria of sequence pattern 3 [sequence pattern 4].

- Control based on two consecutive unlocked joints, whereas in every subsequent step, the position of unlocked joints is mirrored as to the vertical ellipsoid axis [sequence pattern 5].

The particularity of the kinematics of the systems does not allow application of all types of sequence patterns. Specific simulation criteria of the systems are due to the two ground supports that remain unlocked throughout the transformation process. 


\subsection{Sequence Patterns}

Ten sequence patterns $[1,2,3,4,5,-1,-2,-3,-4,-5]$ with four different systems [8-, 9-, 10- and 11-bars] and three different types as to the struts' lengths in relation to the beams $[\mathrm{A}, \mathrm{B}, \mathrm{C}]$ have been investigated as prescribed by the locking and unlocking of the joints according to the previously mentioned constraints. Types $\mathrm{A}, \mathrm{B}$ and $\mathrm{C}$ refer to the joint position as to the overall strut length. Type A refers to a struts' length of $60 \mathrm{~cm}$, type B, $75 \mathrm{~cm}$, and the type $\mathrm{C}, 90 \mathrm{~cm}$ from the inside. In the first sequence pattern, motion based on two consecutive unlocked joints is investigated, and only the 8 and 9-bar systems of types B and $\mathrm{C}$ were appropriate for execution of the transformation steps. Specifically, motion systems [8.C.1, 9.B.1 and 9.C.1] are in position to achieve the transformation steps, independently of any constraint. The second sequence pattern is based on the principle of locking of two consecutive joints and the combinations of unlocked joints refer to the angles respectively. The 8 and 10-bar systems and the motion system 9.A.2 do not succeed in the final joint angle adjustments. The third sequence pattern is feasible and responds to all transformation steps, without flattening of the cables, for the 8- and 10-bar systems of types $\mathrm{C}$ and $\mathrm{A}, \mathrm{B}, \mathrm{C}$ respectively. Suitable types for sequence pattern 4 are the 9- and 10-bar systems of B and C types. Sequence pattern 5 is unsuitable, with exception for the motion systems 9.B.5 and 9.C.5. With regard to the reverse sequences (counter clockwise rotation adjustment) only the motion systems [8.C.-1, 8.C.-3, 9. B.-1, 9.B.-2, 9.C.-1, 9.C.-2, 11.C.-2] are feasible. Main reason for the inefficiency of sequence patterns 4 and 5 is due to the annulment of the adjusted angle in a subsequent step, as well as the flattening of cables while passing through struts. In general, 24 motion systems have proved to succeed the transformation, Fig. 4.

\section{STRUCTURAL TRANSFORMATION RESULTS}

The respective members' responses in the feasible transformations are included in Table 1.

Among the typologies of the 8-bar system, motion system [8.C.-3] develops in all transformation steps the lowest torques at the locked joints, as well as cable's axial forces. Smallest cable's length variation from strut to strut compared to the initial cable's length is observed in motion sequence [8.C.3]. Highest cable's length variation is developed in motion system [8.C.-3].

Lowest bending moments in the locked joints of the 9-bar system and cable's axial forces are developed in motion system [9.B.5]. The motion systems [9.C.-1], [9.C.-2], [9.C.2] and [9.C.1] present small cable's length variation in their transformation.

The 10-bar system distinguishes motion systems [10.B.4], [10.A.4] and [10.C.4] with the lowest torques. Motion systems [10.A.4], [10.B.4] and [10.C.4] have the lowest cable's axial forces, and motion systems [10.C.3], [10.B.3]. The motion system [10.C.4] is distinguished for the lowest cable's length variation.

In the 11-bar system category, only small differentiations are observed, both with regard to the torques and the cable's axial forces, in motion systems [11.C.2] and [11.C.-2]. Lowest values of bending moments, cable's axial forces and length variation are observed in motion system [11.C.-2].

The kinetic analyses of the n-bar systems referred primarily to the typologies design and sequence patterns. Especially, the configuration of the systems in the initial and target position is most significant for the development and completion of the transformation steps of the systems. In the case examples of the current paper, the position of the ellipse in the Cartesian 


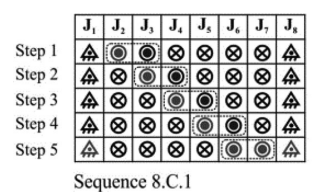

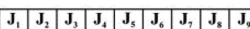

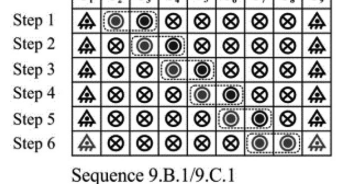

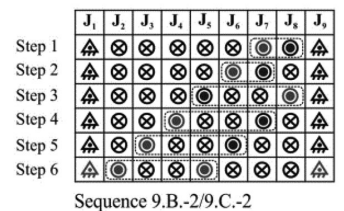

Sequence 9.B.-2/9.C.-2

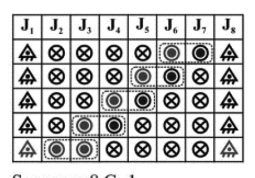

Sequence 8.C.-1

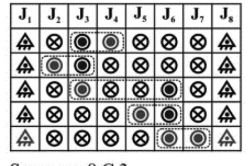

Sequence 8.C.3

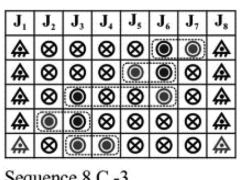

Sequence 8.C.-3

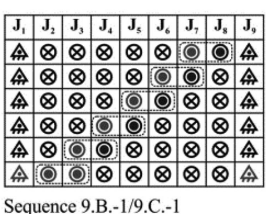

Sequence 9.B.-1/9.C.-1

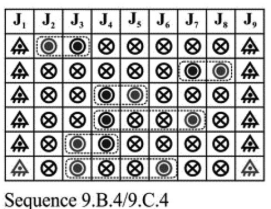

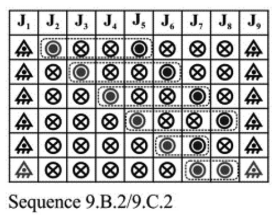

\begin{tabular}{|l|l|l|l|l|l|l|l|l|}
\hline $\mathbf{J}_{\mathbf{1}}$ & $\mathbf{J}_{2}$ & $\mathbf{J}_{3}$ & $\mathbf{J}_{4}$ & $\mathbf{J}_{\mathbf{5}}$ & $\mathbf{J}_{6}$ & $\mathbf{J}_{7}$ & $\mathbf{J}_{\mathbf{8}}$ & $\mathbf{J}_{9}$ \\
\hline
\end{tabular}

\begin{tabular}{|c|c|c|c|c|c|c|c|c|}
\hline$\Delta$ & & () & (2) & $\otimes$ & $\otimes$ & & & $\Delta$ \\
\hline$\Delta$ & $\otimes$ & $\otimes$ & $\otimes$ & $\otimes$ & $\otimes$ & 0 & & $\Delta$ \\
\hline A & $\otimes$ & (0) & (0) & $\otimes$ & $\otimes$ & 8 & & A \\
\hline A & $\otimes$ & Q & (2) & $\otimes$ & 0 & (a) & & $\Delta$ \\
\hline$\Delta$ & Q & (2) & (2) & 0 & $\otimes$ & 8 & & $\Delta$ \\
\hline$\Delta$ & $\otimes$ & $\otimes$ & $\otimes$ & 0 & () & 8 & & $\Delta$ \\
\hline
\end{tabular}

\begin{tabular}{|l|l|l|l|l|l|l|l|l|l|}
\hline $\mathbf{J}_{1}$ & $\mathbf{J}_{2}$ & $\mathbf{J}_{3}$ & $\mathbf{J}_{4}$ & $\mathbf{J}_{\mathbf{s}}$ & $\mathbf{J}_{6}$ & $\mathbf{J}_{1}$ & $\mathbf{J}_{\mathbf{s}}$ & $\mathbf{J}_{9}$ & $\mathbf{J}_{6}$ \\
\hline
\end{tabular} Step $1 \Delta \otimes \otimes O \otimes \otimes O \otimes \otimes \Delta$

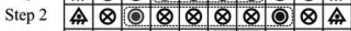

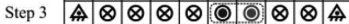

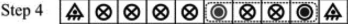

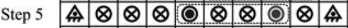

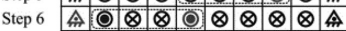

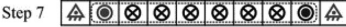

Sequence 10.A.3/10.B.3/10.C.3

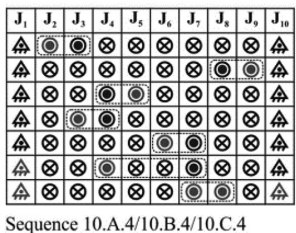

\begin{tabular}{|l|l|l|l|l|l|l|l|l|l|l|}
\hline $\mathbf{J}_{1}$ & $\mathbf{J}_{2}$ & $\mathbf{J}_{3}$ & $\mathbf{J}_{4}$ & $\mathbf{J}_{5}$ & $\mathbf{J}_{6}$ & $\mathbf{J}_{7}$ & $\mathbf{J}_{8}$ & $\mathbf{J}_{9}$ & $\mathbf{J}_{10}$ & $\mathbf{J}_{11}$ \\
\hline
\end{tabular}

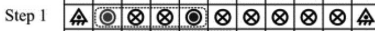

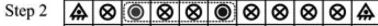

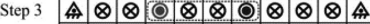

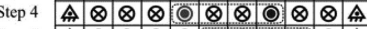

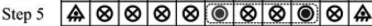
Step $6 \Delta \otimes \otimes \otimes \otimes \otimes 0 \otimes \otimes 0 \Delta$

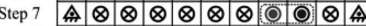

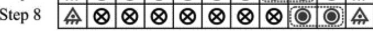
Sequence 11.C.2

\begin{tabular}{|c|c|c|c|c|c|c|c|c|c|c|}
\hline $\mathbf{J}_{1}$ & $\mathbf{J}_{2}$ & $\mathbf{J}_{3}$ & $\mathbf{J}_{4}$ & $\mathbf{J}_{s}$ & $\mathbf{J}_{6}$ & \begin{tabular}{|l|l} 
\\
\end{tabular} & \begin{tabular}{|l|l|} 
\\
\end{tabular} & \begin{tabular}{|l|}
$\mathbf{J}_{9}$ \\
\end{tabular} & $\mid \mathbf{J}_{10}$ & $J_{11}$ \\
\hline 命 & $\otimes$ & $\otimes$ & $\otimes$ & (8) & $\otimes$ & 0 & $\otimes$ & $\otimes$ & () & 命 \\
\hline A & $\otimes$ & $\otimes$ & $\otimes$ & $\otimes$ & 0 & $\otimes$ & $\otimes$ & 0 & $\otimes$ & 命 \\
\hline \begin{tabular}{|l|} 
A \\
\end{tabular} & $\otimes$ & $\otimes$ & Q & (0) & $\otimes$ & $\otimes$ & 0 & $\otimes$ & $\otimes$ & $\Delta$ \\
\hline 命 & (2) & $\otimes$ & 0 & $\otimes$ & $\otimes$ & 0 & Q & (2) & $\otimes$ & 命 \\
\hline 命 & $\otimes$ & 0 & $\otimes$ & $\otimes$ & 0 & $\otimes$ & $\otimes$ & $\otimes$ & $\otimes$ & 命 \\
\hline 侖 & 0 & $\otimes$ & $\otimes$ & (0) & (2) & $\otimes$ & $\otimes$ & $\otimes$ & $\otimes$ & $\Delta$ \\
\hline A & Q & 0 & (2) & Q & (2) & $\otimes$ & $\otimes$ & Q & $\otimes$ & $\Delta$ \\
\hline 命 & (6) & (2) & (2) & Q & (2) & Q & $\otimes$ & Q & $\otimes$ & $\Delta$ \\
\hline
\end{tabular}

Figure 4: Feasible control sequences for implementing the required shape adjustment $(\otimes$ : locked joint, $\odot:$ unlocked joint, $\Delta$ : pivoted-to-the-ground joint, shaded symbols represent the currently adjusted joints).

system of coordinates influences the possibilities of kinematic solutions. By shifting the centre of ellipse upwards along the $\mathrm{z}$-axis, the feasible transformation solutions increase.

The complete system transformation and achievement of the target position as to the number of bars composing the systems has been most successful with application of 9 bars with 12 feasible types, followed by the application of 10, 8, and 11 bars, with six, four and two feasible types, respectively. Principally, application of the 8- and 11-bar systems led to large angles at the initial position, and did not enable further adjustment.

The selection of feasible motion systems in relation to the systems with 8, 9, 10 and 11 bars, based on the specific criteria set in the analyses, distinguishes the most favourable motion systems [8.C.-3], [9.B.5], [10.A.4] and [11.C.-2], with regard to their performance from the initial to the target position. Specifically, the comparison of bending moments, axial forces and cable's length variation as to the motion systems is not self-evident, since it also depends from the struts' position and the sequence patterns. 
Table 1: System locked joints' maximum torques $\left(\mathrm{J}_{\mathrm{i}, \mathrm{max}}\right)$, cables' maximum axial forces $\left(\mathrm{A}_{\mathrm{i}, \max }\right)$ and variation length $\left(\Delta \mathrm{l}_{\max }\right)$.

\begin{tabular}{|c|c|c|c|}
\hline Motion Systems & $\begin{array}{l}\mathrm{J}_{\mathrm{i}, \max } \\
{[\mathrm{kNm}]}\end{array}$ & $\begin{array}{l}\mathrm{A}_{\mathrm{i}, \max } \\
{[\mathrm{kN}]}\end{array}$ & $\begin{array}{l}\Delta \mathrm{l}_{\max } \\
{[\mathrm{cm}]}\end{array}$ \\
\hline 8.C.1 & 9,17 & 5,75 & 37 \\
\hline 8.C. -1 & 8,33 & 12,90 & 34 \\
\hline 8.C. 3 & 13,44 & 6,71 & 28 \\
\hline 8.C. -3 & 7,10 & 5,15 & 52 \\
\hline 9.B.1 & 8,23 & 4,88 & 54 \\
\hline 9.B.-1 & 9,12 & 5,29 & 44 \\
\hline 9.B.2 & 11,63 & 10,90 & 41 \\
\hline 9.B. -2 & 17,03 & 15,36 & 51 \\
\hline 9.B.4 & 13,96 & 12,15 & 53 \\
\hline 9.B.5 & 8,27 & 3,89 & 44 \\
\hline 9.C.1 & 11,18 & 9,90 & 16 \\
\hline 9.C. -1 & 17,01 & 3,86 & 11 \\
\hline 9.C.2 & 18,54 & 14,07 & 16 \\
\hline 9.C.-2 & 19,02 & 14,09 & 14 \\
\hline 9.C.4 & 15,39 & 10,95 & 30 \\
\hline 9.C.5 & 9,37 & 3,85 & 23 \\
\hline 10.A.3 & 16,74 & 9,93 & 15 \\
\hline 10.A.4 & 9,68 & 4,95 & 19 \\
\hline 10.B.3 & 10,94 & 8,93 & 63 \\
\hline 10.B.4 & 9,69 & 4,68 & 48 \\
\hline 10.C.3 & 15,69 & 8,45 & 52 \\
\hline 10.C.4 & 9,97 & 4,68 & 16 \\
\hline 11.C. 2 & 18,09 & 15,98 & 10 \\
\hline 11.C. -2 & 17,87 & 15,03 & 12 \\
\hline
\end{tabular}

\section{CONCLUSIONS}

Kinetic hybrid systems, composed of customized hinge-connected members strengthened through a secondary system of struts and continuous cables with variable length, have been developed and investigated in their kinetic behaviour. The systems succeed in morphological transformation through reconfiguration based on application of the effective 4-bar control concept and the dual operation of the cables in providing stability and kinetic activation. Energy related advantages of this approach result from the minimization of the number of actuators and their implementation on the supports only in avoiding any related self-weight increase of the primary systems. The kinetic behaviour of the systems has been investigated 
in simulation of 24 feasible motion systems of different sequence patterns and geometrical characteristics. The analysis has proven that the configuration of the systems and members plays a significant role in the motion planning. In this respect, smaller angles at the initial systems' position, as applied in the 9-bar system, are more effective for reconfiguration and prevention of flattening of the beams. The analysis concluded with most favourable motion systems with regard to their kinetic performance. In this respect, future work will involve further experimental investigation of this concept and verification of the simulation results obtained.

\section{REFERENCES}

[1] Pellegrino, S. (ed), Deployable Structures, International Center for Mechanical Sciences, CISM courses and lectures, 412, 2001.

[2] Gantes, C.J., Deployable Structures: Analysis and Design, WIT Press: Southampton, 2001.

[3] Hanaor, A., Tensegrity: Theory and Application. Beyond the Cube, ed. J.F. Gabriel, John Wiley \& Sons, pp. 385-408, 1997.

[4] Tibert, G., Deployable Tensegrity Structures for Space Applications. Ph.D. Thesis, Royal Institute of Technology, Stockholm, pp. 1-30, 2002.

[5] Adam, B. \& Smith, I.F.C., Active tensegrity: a control framework for an adaptive civilengineering structure. Computers and Structures, 86(23-24), pp. 2215-2223, 2008. http://dx.doi.org/10.1016/j.compstruc.2008.05.006

[6] Arsenault, M. \& Gosselin, C., Kinematic, static and dynamic analysis of a planar 1-dof tensegrity mechanism. Journal of Mechanical Design, 127(6), pp. 1152-1160, 2005. http://dx.doi.org/10.1115/1.1913705

[7] Arsenault, M. \& Gosselin, C., Kinematic, static and dynamic analysis of a planar 2-dof tensegrity mechanism. Mechanism and Machine Theory, 41(9), pp. 1072-1089, 2006. http://dx.doi.org/10.1016/j.mechmachtheory.2005.10.014

[8] Arsenault, M. \& Gosselin, C., Kinematic, static and dynamic analysis of a spatial 3-DOF tensegrity mechanism. Journal of Mechanical Design, 128(5), pp. 1061-1069, 2006. http://dx.doi.org/10.1115/1.2218881

[9] Arsenault, M. \& Gosselin, C., Kinematic and static analysis of a 3-PUPS spatial tensegrity mechanism. Mechanism and Machine Theory, 44(1), pp. 162-179, 2009. http://dx.doi.org/10.1016/j.mechmachtheory.2008.02.005

[10] Bel Hadj Ali, N., Rhode-Barbarigos, L. \& Smith, I.F.C., Analysis of clustered tensegrity structures using a modified dynamic relaxation algorithm. International Journal of Solids and Structures, 48(5), pp. 637-647, 2011. http://dx.doi.org/10.1016/j.ijsolstr.2010.10.029

[11] Moored, K.W., Kemp, T.H., Hole, N.E. \& Bart-Smith, H., Analytical predictions, optimization and design of a tensegrity-based artificial pectoral fin. International Journal of Solids and Structures, 48(22-23), pp. 3142-3159, 2011.

http://dx.doi.org/10.1016/j.ijsolstr.2011.07.008

[12] Rhode-Barbarigos, L., An Active Deployable Structure. Ph.D. Thesis, EPFL, Lausanne, 2012.

[13] Pinero, E.P., Project for mobile theatre. Architectural Design, 12(1), pp. 154-155, 1961.

[14] Escrig, F., Expandable space structures. International Journal of Space Structures, 1(2), pp. 79-91, 1985. 
[15] Escrig, F. \& Valcarcel, J.P., Curved expandable space grids. Proceedings of International Conference on the Design and Construction of Non-Conventional Structures, London, pp. 157-168, 1987. http://dx.doi.org/10.4203/ccp.7.18.1

[16] Hoberman, C., Unfolding architecture: an object that is identically a structure and a mechanism. Architectural Design, 63(1), pp. 56-59, 1993.

[17] You, Z. \& Pellegrino, S., Foldable bar structures. International Journal of Solids and Structures, 34(15), pp. 1825-1847, 1997. http://dx.doi.org/10.1016/S0020-7683(96)00125-4

[18] Akgün, Y., Gantes, C.J., Kalochairetis, K. \& Kiper G., A novel concept of convertible roofs with high transformability consisting of planar scissor-hinge structures. Engineering Structures, 32(9), pp. 2873-2883, 2010.

http://dx.doi.org/10.1016/j.engstruct.2010.05.006

[19] Akgün, Y., Gantes, C.J., Sobek, W., Korkmaz, K. \& Kalochairetis, K., A novel adaptive spatial scissor-hinge structural mechanism for convertible roofs. Engineering Structures, 33(4), pp. 1365-1376, 2011.

http://dx.doi.org/10.1016/j.engstruct.2011.01.014

[20] Phocas, M.C., Kontovourkis, O. \& Matheou, M., Kinetic hybrid structure development and simulation. International Journal of Architectural Computing, 10(1), pp. 67-86, 2012. http://dx.doi.org/10.1260/1478-0771.10.1.67

[21] Christoforou, E.G., Mueller, A., Phocas, M.C., Matheou, M. \& Arnos, S., Design and control concept for reconfigurable architecture. Journal of Mechanical Design, 137(4), p.042302, 2015.

http://dx.doi.org/10.1115/1.4029617

[22] Phocas, M.C., Christoforou, E.G. \& Matheou, M., Design and motion planning of a reconfigurable hybrid structure. Engineering Structures, 101(29), pp. 376-385, 2015. http://dx.doi.org/10.1016/j.engstruct.2015.07.036 